\title{
Clinical features in different age groups of patients with autoimmune hepatitis
}

\author{
MILIN PENG, YI LI, MIN ZHANG, YONGFANG JIANG, YUAN XU, YI TIAN, \\ FENG PENG and GUOZHONG GONG
}

Center of Liver Diseases, Second Xiangya Hospital, Central South University, Changsha, Hunan 410011, P.R. China

Received June 17, 2013; Accepted October 17, 2013

DOI: $10.3892 /$ etm.2013.1363

\begin{abstract}
The Chinese population are at an increased risk of autoimmune hepatitis (AIH). The aims of this study were to determine the demographic and clinical features of AIH in China. A total of 83 patients with AIH diagnosed by the revised scoring system were re-analyzed, and the clinical presentations among the different ages were compared. The patients were classified according to age at presentation. AIH occurred in patients aged $\leq 30$ years $(9.6 \%), 31-39$ years $(10.8 \%)$, $40-49$ years $(16.9 \%), 50-59$ years $(31.3 \%)$ and $\geq 60$ years $(31.3 \%)$. There were no differences in the form of the clinical presentation, concurrent autoimmune diseases, cirrhosis distribution and autoantibodies among the groups. However, patients aged $\geq 60$ years presented with higher levels of alkaline phosphatase (ALP) and $\gamma$-glutamyl transpeptidase $(\gamma$-GT) compared with patients aged $\leq 30$ years $(\mathrm{P}=0.034, \mathrm{P}=0.043$, respectively), and patients aged 31-39 years had a significantly lower immunoglobulin $\mathrm{G}(\mathrm{IgG})$ level compared with those aged 50-59 years $(\mathrm{P}=0.049)$ and those aged $\geq 60$ years $(\mathrm{P}=0.012)$. By contrast, patients aged $\leq 30$ years had a significantly higher total bilirubin (TBIL) level compared with those aged 31-39 years $(\mathrm{P}=0.007), 50-59$ years $(\mathrm{P}=0.002)$ and $\geq 60$ years $(\mathrm{P}=0.013)$. $\mathrm{A}$ substantial portion of patients with $\mathrm{AIH}$ were aged $>60$ years, indicating a poor liver-associated outcome under current management strategies. Elderly patients appeared to be more asymptomatic compared with the younger patients.
\end{abstract}

\section{Introduction}

Autoimmune hepatitis (AIH) is an unknown cause of liver inflammation, and the diagnosis of AIH requires the presence of characteristic clinical and laboratory features, and histological abnormalities (1). The incidence of AIH among

Correspondence to: Professor Guozhong Gong, Center of Liver Diseases, Second Xiangya Hospital, Central South University, 139 Renmin Zhong Road, Changsha, Hunan 410011, P.R. China E-mail: 18647110035@163.com

Key words: autoimmune hepatitis, clinical presentation, concurrent autoimmune diseases, China white, Northern Europeans is 1.9 cases per 100,000 people per year, and its point prevalence is 16.9 cases per 100,000 people per year (2). In the USA, AIH affects between 100,000 and 200,000 individuals (3), and it accounts for 6\% of liver transplantations (4). The frequency of AIH among patients with chronic liver disease in North America is between 11 and 23\% (5). AIH should be considered in all individuals with acute and chronic hepatitis with an undetermined cause (6). Clinical presentations of the disease are variable, ranging from asymptomatic abnormal liver enzymes to fulminant liver failure or advanced decompensated cirrhosis (7). The frequency of cirrhosis at presentation is $>30 \%$ (8). Concurrent immune disorders may mask the diagnosis of AIH. The diagnostic criteria and scoring system for AIH were codified by an international panel in 1993 (10) and revised in 1999 (1).

In comparison with the age- and gender-matched general population, with effective treatments available the mortality of AIH is two-fold higher than that of the general population $(11,12)$. A long-term study reported that only $\sim 40 \%$ of patients with AIH achieve complete remission (13). Traditionally, it was considered that clinical and demographic factors were closely associated with a poor outcome. Studies have reported that age distribution, serum aspartate aminotransferase (AST), alanine aminotransferase (ALT) and serum albumin levels were significant predictors of liver-associated mortality or liver transplantation $(14,15)$. The majority of the current studies regarding the clinical characteristics associated with a poor outcome have focused on European and Japanese patients. Therefore, it is important to identify the clinical manifestations found in Chinese patients with $\mathrm{AIH}$ at various ages.

\section{Patients and methods}

Study population. Patients with AIH who were admitted to the Second Xiangya Hospital (Changsha, China) between 2002 and 2013, and who had complete clinical, laboratory and histological data, were enrolled in this retrospective study. The diagnoses were based on the 1999 revised criteria of the International Group of Autoimmune Hepatitis (1). Liver biopsy results were included if available, and two hepatopathologists reviewed all liver tissue specimens. Those patients with insufficient data for the diagnosis of AIH prior to treatment and other liver diseases [viral hepatitis, Wilson's disease, non-alcoholic 
fatty liver disease (NFLD), primary biliary cirrhosis (PBC) and primary sclerosing cholangitis (PSC)] were excluded from this study. Patients were categorized according to their age at presentation into the following groups: $\leq 30$ years, 31-39 years, $40-49$ years, $50-59$ years and $\geq 60$ years. This study was approved by The Ethics Committee of the Second Xiangya Hospital of Central South University (Changsha, China). Informed consent was obtained from all patients.

Clinical and laboratory assessments. Clinical examinations and conventional laboratory tests were assessed prior to providing any specific therapy to the patients. The presence of other concurrent autoimmune diseases was also investigated. Liver function tests were performed, including the measurements of the serum levels of alkaline phosphatase (ALP), $\gamma$-glutamyl transpeptidase $(\gamma$-GT), AST, ALT, total bilirubin (TBIL) and serum immunoglobulin G (IgG), by performing immunonephelometry on all patients. Smooth muscle antibodies (SMA), antinuclear antibodies (ANA), perinuclear antineutrophil cytoplasmic antibodies (pANCA), antimitochondrial antibodies (AMA), antibodies to liver kidney microsome type 1 (LKM1) and soluble liver antigen/liver-pancreas antigen (SLA/LP) were evaluated using indirect immunofluorescence. Each patient was seronegative for AMA.

Statistical analysis. A descriptive analysis, including means, standard deviation and frequencies, is presented. An independent sample t-test was used to assess the correlation between continuous variables and the $\chi^{2}$ test was used for the analysis of categorical variables. $\mathrm{P}<0.05$ was considered in indicate a statistically significant difference. The SPSS statistical software version 17.0 (SPSS, Inc., Chicago, IL, USA) was used for all statistical analyses.

\section{Results}

Study population. A total of 83 Chinese patients fulfilled the 1999 revised criteria of the International Group of Autoimmune Hepatitis; 41 patients (49.4\%) were classified as 'definite' AIH and $42(50.6 \%)$ were classified as 'probable' AIH. At presentation, the patients were graded according to their age. Patients aged $\leq 30$ years $(9.6 \%), 31-39$ years $(10.8 \%), 40-49$ years (16.9\%), $50-59$ years $(31.3 \%)$ and $\geq 60$ years $(31.3 \%)$ with AIH were enrolled in the study. In total, 26 patients (31.3\%) were $\geq 60$ years old. The mean age of this group (the elderly population) was 65.62 \pm 4.92 years (range, 60-78 years) and the male to female ratio was $3: 23$. In total, 26 patients (31.3\%) were aged between 50 and 59 years. The mean age was $54.65 \pm 2.67$ years and the male to female ratio was 2:24. A total of eight patients $(9.6 \%)$ constituted the young population, with the mean age of $21.38 \pm 6.74$ years (range, 10-30 years). The disease frequency in each age range increased $>40$ years (Fig. 1), and the peak occurrences were in the patients aged 50-59 years and in the elderly patients. Each group was similar with regard to gender distribution.

Clinical presentation. The clinical manifestations of AIH are unspecific, and other chronic liver diseases may have the same symptoms. Anorexia, fatigue and jaundice were the

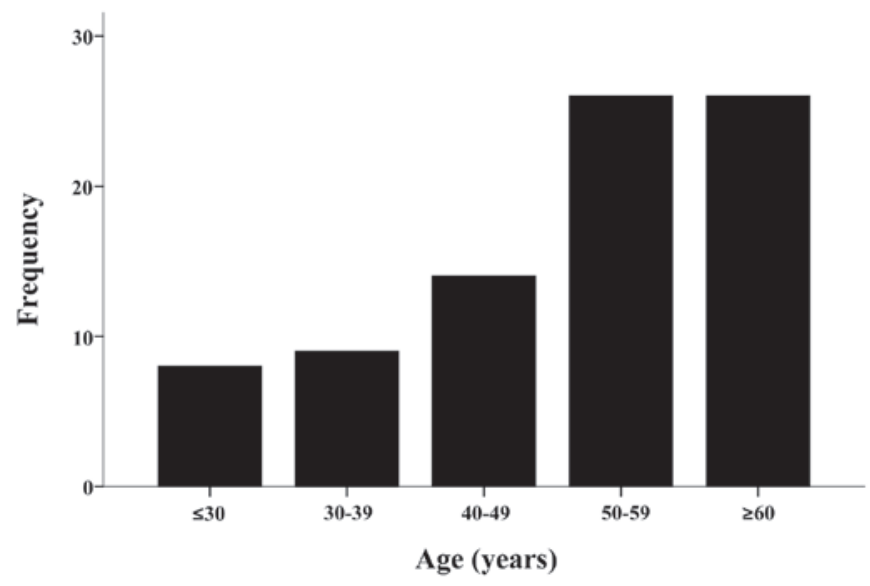

Figure 1. Age distribution of autoimmune hepatitis in 83 Chinese patients.

most common symptoms observed in $>70 \%$ of the 83 patients. Furthermore, $15 \%$ presented with a fever and $\sim 10 \%$ of patients had pruritus. Overall, $76.9 \%$ of patients presented with up to three symptoms, and $23.1 \%$ presented with four symptoms. Livercirrhosis was diagnosed in $10(12 \%)$ patients, among whom five patients (50\%) were between the ages of 50 and 59 years and three patients (30\%) were elderly. However, the frequency of cirrhosis was not statistically different between the elderly patients ( $\geq 60$ years old) and the young patients ( $\leq 30$ years old) $(\mathrm{P}>0.05)$.

Laboratory data. The mean serum levels of liver function are illustrated in Table I. Patients aged $\leq 30$ years $(136.53 \pm 74.03 \mathrm{U} / 1$, $\mathrm{P}=0.034)$ and aged $40-49$ years $(147.43 \pm 52.71 \mathrm{U} / 1, \mathrm{P}=0.021)$ presented with significantly lower levels of of ALP than patients aged $\geq 60$ years $(238.78 \pm 163.29$ U/l). Patients aged $\leq 30$ years $(119.71 \pm 145.92 \mathrm{U} / \mathrm{l})$ also presented with significantly lower $\gamma$-GT levels compared with patients aged 50-59 years $(257.69 \pm 175.56 \mathrm{U} / 1, \mathrm{P}=0.037)$ and those aged $\geq 60$ years (253.27 $\pm 193.96 \mathrm{U} / 1, \mathrm{P}=0.043)$. Similarly, patients aged 31-39 years $(18.74 \pm 5.83 \mathrm{~g} / \mathrm{l})$ presented with significantly lower IgG levels than those aged 50-59 years $(25.41 \pm 6.05 \mathrm{~g} / \mathrm{l}$, $\mathrm{P}=0.049)$ and those aged $\geq 60$ years $(27.29 \pm 11.33 \mathrm{~g} / \mathrm{l}, \mathrm{P}=0.012)$. By contrast, patients aged $\leq 30$ years $(351.33 \pm 284.94 \mu \mathrm{mol} / \mathrm{l})$ had significant higher TBIL levels compared with those aged 31-39 years $(116.46 \pm 106.09 \mu \mathrm{mol} / 1, \mathrm{P}=0.007)$, those aged 50-59 years $(125.28 \pm 112.43 \mu \mathrm{mol} / \mathrm{l}, \mathrm{P}=0.002)$ and those aged $\geq 60$ years $(172.83 \pm 33.82 \mu \mathrm{mol} / 1, \mathrm{P}=0.013)$.

In total, 51 of the 83 patients were positive for ANA with a titer of $\geq 1: 40$. Among the elderly patients, 19 were positive for ANA, while among the patients aged 50-59 years and $\leq 30$ years, 17 and five patients were positive for ANA, respectively. However, there were no differences in the positive proportions of ANA among each group (Table I).

Concurrent autoimmune diseases. Of the 26 elderly patients, $10(59 \%)$ presented with coexisting autoimmune diseases, including four patients with Sjögren's Syndrome, five patients with systemic lupus erythematosus and one patient with ulcerative colitis. Similarly, 11 of the 26 patients aged 50-59 years presented with concurrent autoimmune diseases (four patients with Sjögren's Syndrome, two with autoimmune thyroiditis 
Table I. Clinical features of 83 Chinese patients with autoimmune hepatitis in each group.

\begin{tabular}{|c|c|c|c|c|c|}
\hline \multirow[b]{2}{*}{ Features } & \multicolumn{5}{|c|}{ Age category (years) $(n=83)$} \\
\hline & $\leq 30(n=8)$ & $31-39(n=9)$ & $40-49(n=14)$ & $50-59(n=26)$ & $\geq 60(n=26)$ \\
\hline Female (n) & 5 & 6 & 13 & 24 & 23 \\
\hline Age (years) & $21.3 \pm 6.7$ & $37.4 \pm 1.9$ & $45.9 \pm 2.8$ & $54.6 \pm 2.6$ & $65.6 \pm 4.9$ \\
\hline Cirrhosis (n) & 1 & 1 & 0 & 5 & 3 \\
\hline $\operatorname{ALT}(\mathrm{U} / \mathrm{l})$ & $151.8 \pm 133.7$ & $312.2 \pm 338.4$ & $288.5 \pm 233.5$ & $384.2 \pm 397.7$ & $287.9 \pm 288.4$ \\
\hline $\operatorname{AST}(\mathrm{U} / \mathrm{l})$ & $193.6 \pm 147.9$ & $422.9 \pm 596.9$ & $353.0 \pm 314.1$ & $385.7 \pm 367.1$ & $310.6 \pm 213.1$ \\
\hline TBIL $(\mu \mathrm{mol} / \mathrm{l})$ & $351.3 \pm 284.9$ & $116.5 \pm 106.1$ & $203.5 \pm 224.9$ & $125.3 \pm 112.4$ & $172.2 \pm 172.8$ \\
\hline $\operatorname{ALP}(\mathrm{U} / \mathrm{l})$ & $136.5 \pm 74.0$ & $187.5 \pm 138.5$ & $147.4 \pm 52.7$ & $191.2 \pm 83.4$ & $238.8 \pm 163.3$ \\
\hline$\gamma$-GT (U/l) & $119.7 \pm 145.9$ & $161.5 \pm 95.1$ & $135.8 \pm 81.7$ & $257.7 \pm 175.6$ & $253.3 \pm 194.0$ \\
\hline $\operatorname{IgG}(\mathrm{g} / \mathrm{l})$ & $25.1 \pm 11.4$ & $18.7 \pm 5.8$ & $22.1 \pm 6.1$ & $25.4 \pm 6.1$ & $27.3 \pm 11.3$ \\
\hline ANA $(\geq 1: 40)$ & 5 & 3 & 7 & 17 & 19 \\
\hline
\end{tabular}

ALT, alanine aminotransferase; AST, aspartate aminotransferase; TBIL, total bilirubin; ALP, alkaline phosphatase; $\gamma$-GT, $\gamma$-glutamyl transpeptidase; IgG, immunoglobulin G; ANA, antinuclear antibodies.

and rheumatoid arthritis, and three with systemic lupus erythematosus). Five of the eight younger patients (aged $\leq 30$ years) presented with concurrent autoimmune diseases (two patients with autoimmune thyroiditis and systemic lupus erythematosus, and one patient with rheumatoid arthritis). However, the distribution of concurrent autoimmune diseases in each age group was not statistically significant $(\mathrm{P}=0.398)$.

\section{Discussion}

In China, the etiology of chronic hepatitis is mainly hepatitis virus infection. Since the recognition of autoimmune liver diseases, these diseases have become one of the major forms of non-viral chronic liver diseases in China. Studies on the prevalence of Chinese patients with $\mathrm{AIH}$ are rare.

The present study investigated the differences associated with age in the clinical and laboratory features of Chinese patients with AIH diagnosed using the revised 1999 scoring system (1). In this study, the age of onset in Chinese patients showed that the peak occurrences of AIH exist in the age groups of 50-59 years and $\geq 60$ years, and there are few young patients $(9.6 \%)$ with AIH. The proportion of young patients is smaller than in previous studies regarding Japanese patients. The frequency of liver cirrhosis at first diagnosis of AIH was $<12 \%$, lower than other studies where cirrhosis at presentation was detected and reported in $27-42 \%$ of patients $(16,17)$.

The present study demonstrated that elderly patients ( $\geq 60$ years old) presented with higher serum ALP and $\gamma$-GT levels compared with younger patients ( $\leq 30$ years old). By contrast, the young patients presented with higher TBIL levels compared with elderly patients. This result indicated that patients aged $\leq 30$ years may have more severe disease activity than elderly patients. A previous study from Japan demonstrated that elderly patients presented with more asymptomatic clinical features compared with the young patients (17). The results of the present study also suggested that elderly patients may be misdiagnosed in the early stage due to the fact that they may not exhibit any symptoms. This may be one of the reasons for the prevalence of autoimmune diseases and the increased presentation in elderly patients compared with younger patients in China. However, the occurrence of cirrhosis in each group was similar in the present study, and the rate was markedly lower compared with groups of patients from North America (16) and Japan (17).

$\mathrm{AIH}$ is associated with a number of distinct circulating autoantibodies. The most common autoantibodies are ANA and SMA. It was revealed that $96 \%$ of adult patients with AIH from North America had ANA and SMA (18). Anti-SLA/LP and pANCA may be useful in identifying patients with AIH from those who lack typical serological findings $(19,20)$. Anti-SLA/LP are highly specific markers of AIH and more commonly found in association with the conventional autoantibodies; however, they also are occasionally found in patients with AIH who are negative for ANA, SMA, and anti-LKM1 (19). It has been reported that anti-SLA are highly specific for the diagnosis of autoimmune liver disease, and their detection may reveal patients with a more severe degree of the disease and a worse predicted outcome (21). pANCA are non-specific, but are commonly present. They have been used to reclassify patients with cryptogenic chronic hepatitis, such as AIH; however, they have not been formally assimilated into the diagnostic algorithm (20). In the present study, we observed that ANA was the most common autoantibody in Chinese patients with AIH. Among these Chinese patients with AIH, $61.4 \%$ were ANA-positive. SLA/LP (14.5\%) was another common autoantibody. Serum levels of AST and IgG were found to be higher in ANA-positive patients; however, there was no statistically significant difference between patients who were positive and negative for ANA, which was similar to a previous Japanese study (22).

Concurrent immune disorders may mask the underlying liver disease. Autoimmune thyroiditis, Graves' disease, synovitis and ulcerative colitis are the most common immune-mediated disorders associated with $\mathrm{AIH}$ in adults from North America (23). Notably, concurrent immune diseases were more commonly observed in this group of 
Chinese patients with AIH (42.2\%) than in the group of patients from the west (24). It has been reported that associated autoimmune diseases were observed in $15-34 \%$ of patients from the West (24). In the present study it was observed that Sjögren's Syndrome was the most commonly associated autoimmune disorder. According to a nationwide survey in Japan (22), the prevalence of complicating Sjögren's syndrome in patients with AIH was $\sim 10 \%$. Czaja and Carpenter (16) reported that elderly patients presented with concurrent thyroid and rheumatic diseases more often than patients aged $\leq 30$ years. Certain studies have suggested that human leukocyte antigen DR4 occurred more frequently in elderly patients. However, in the present study, autoimmune thyroiditis was uncommon in elderly Chinese patients. The distribution of the human leukocyte antigen is not fully recognized in China, and further studies are required to analyze the human leukocyte antigen in large number of patients with AIH.

A long-term study established that incomplete normalization of ALT at 6 months, low serum albumin concentration at diagnosis, and an age at presentation of $<20$ years or $>60$ years were significant independent predictors of a poor outcome (15). However, histological cirrhosis at diagnosis was not associated with a poor prognosis and did not affect the response to initial immunosuppressive treatment. In the present study, a considerable portion of patients with AIH were aged $>60$ years, indicating a poor liver-associated outcome under current management strategies. In addition, even when treated with the usual management strategy, there is an increased risk that extra-hepatic malignancy, such as skin (non-melanoma) and hematological cancers, may be induced by immunosuppressive therapy. Further studies should explore effective ways to improve the survival of patients with AIH without increasing the risk of extra-hepatic malignancy.

One of the limitations of the present study was the relatively small number of patients; multicenter national data are required. Moreover, the detection of HLA-DR3 and DR4 is rare in China, and the results from the studies performed in other countries have demonstrated that HLA status may affect clinical manifestations, behavior and the outcome of treatment (16).

In conclusion, a substantial portion of patients with $\mathrm{AIH}$ were $>60$ years old, indicating a poor liver-associated outcome under current management strategies. Elderly patients exhibited higher levels of ALP and $\gamma$-GT, and a lower TBIL level than younger patients. Chinese patients with AIH had a lower frequency of cirrhosis at presentation and a higher frequency of concurrent autoimmune diseases compared with patients from Europe, North America and Japan. However, the frequencies of cirrhosis, concurrent autoimmune disease and autoantibodies were similar among each group.

\section{References}

1. Alvarez F, Berg PA, Bianchi FB, et al: International Autoimmune Hepatitis Group Report: review of criteria for diagnosis of autoimmune hepatitis. J Hepatol 31: 929-938, 1999.
2. Seo S, Toutounjian R, Conrad A, et al: Favorable outcomes of autoimmune hepatitis in a community clinic setting. J Gastroenterol Hepatol 23: 1410-1414, 2008.

3. Czaja AJ: Autoimmune liver disease. Curr Opin Gastroenterol 19: 232-242, 2003

4. Wiesner RH, Demetris AJ, Belle SH, et al: Acute allograft rejection: incidence, risk factors, and impact on outcome. Hepatology 28: 638-645, 1998.

5. Feld JJ, Dinh H, Arenovich T, et al: Autoimmune hepatitis: effect of symptoms and cirrhosis on natural history and outcome. Hepatology 42: 53-62, 2005.

6. Czaja AJ: Diverse manifestations and evolving treatments of autoimmune hepatitis. Minerva Gastroenterol Dietol 51: 313-333, 2005.

7. Floreani A, Niro G, Rosa Rizzotto E, et al: Type I autoimmune hepatitis: clinical course and outcome in an Italian multicentre study. Aliment Pharmacol Ther 24: 1051-1057, 2006.

8. Parker DR and Kingham JG: Type I autoimmune hepatitis is primarily a disease of later life. QJM 90: 289-296, 1997.

9. Ichai P, Duclos-Vallée JC, Guettier C, et al: Usefulness of corticosteroids for the treatment of severe and fulminant forms of autoimmune hepatitis. Liver Transpl 13: 996-1003, 2007.

10. Johnson PJ and McFarlane IG: Meeting report: International Autoimmune Hepatitis Group. Hepatology 18: 998-1005, 1993.

11. Ngu JH, Gearry RB, Frampton CM, et al: Mortality and the risk of malignancy in autoimmune liver diseases: a population-based study in Canterbury, New Zealand. Hepatology 55: 522-529, 2012.

12. Hoeroldt B, McFarlane E, Dube A, et al: Long-term outcomes of patients with autoimmune hepatitis managed at a nontransplant center. Gastroenterology 140: 1980-1989, 2011.

13. Manns MP, Woynarowski M, Kreisel W, et al: Budesonide induces remission more effectively than prednisone in a controlled trial of patients with autoimmune hepatitis. Gastroenterology 139: 1198-1206, 2010

14. Al-Chalabi T, Underhill JA, Portmann BC, et al: Effects of serum aspartate aminotransferase levels in patients with autoimmune hepatitis influence disease course and outcome. Clin Gastroenterol Hepatol 6: 1389-1395, 2008.

15. Ngu JH, Gearry RB, Frampton CM and Stedman CA: Predictors of poor outcome in patients with autoimmune hepatitis: A population-based study. Hepatology 57: 2399-2406, 2013.

16. Czaja AJ and Carpenter HA: Distinctive clinical phenotype and treatment outcome of type 1 autoimmune hepatitis in the elderly. Hepatology 43: 532-538, 2006.

17. Miyake Y, Iwasaki Y, Takaki A, et al: Clinical features of Japanese elderly patients with type 1 autoimmune hepatitis. Intern Med 46: 1945-1949, 2007.

18. Czaja AJ: Behavior and significance of autoantibodies in type 1 autoimmune hepatitis. J Hepatol 30: 394-401, 1999.

19. Baeres M, Herkel J, Czaja AJ, et al: Establishment of standardised SLA/LP immunoassays: specificity for autoimmune hepatitis, worldwide occurrence, and clinical characteristics. Gut 51: 259-264, 2002.

20. Zauli D, Ghetti S, Grassi A, et al: Anti-neutrophil cytoplasmic antibodies in type 1 and type 2 autoimmune hepatitis. Hepatology 25: 1105-1107, 1997.

21. Czaja AJ, Donaldson PT and Lohse AW: Antibodies to soluble liver antigen/liver pancreas and HLA risk factors for type 1 autoimmune hepatitis. Am J Gastroenterol 97: 413-419, 2002.

22. Toda G, Zeniya M, Watanabe F, et al: Present status of autoimmune hepatitis in Japan - correlating the characteristics with international criteria in an area with a high rate of $\mathrm{HCV}$ infection. Japanese National Study Group of Autoimmune Hepatitis. J Hepatol 26: 1207-1212, 1997.

23. Czaja AJ and Freese DK; American Association for the Study of Liver Disease: Diagnosis and treatment of autoimmune hepatitis. Hepatology 36: 479-497, 2002.

24. Krawitt EL: Autoimmune hepatitis. N Engl J Med 354: 54-66, 2006. 